\title{
Normal Values of Magnetic Relaxation Parameters of Spine Components with the Synthetic MRI Sequence
}

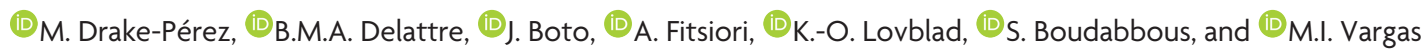

\begin{abstract}
BACKGROUND AND PURPOSE: SyMRI is a technique developed to perform quantitative MR imaging. Our aim was to analyze its potential use for measuring relaxation times of normal components of the spine and to compare them with values found in the literature using relaxometry and other techniques.
\end{abstract}

MATERIALS AND METHODS: Thirty-two spine MR imaging studies (10 cervical, 5 dorsal, 17 lumbosacral) were included. A modified multiple-dynamic multiple-echo sequence was added and processed to obtain quantitative $\mathrm{Tl}$ (millisecond), $\mathrm{T} 2$ (millisecond), and proton density (percentage units [pu]) maps for each patient. An ROI was placed on representative areas for CSF, spinal cord, intervertebral discs, and vertebral bodies, to measure their relaxation.

RESULTS: Relaxation time means are reported for CSF ( $T 1=4273.4 \mathrm{~ms} ; \mathrm{T} 2=1577.6 \mathrm{~ms}$; proton density $=107.5 \mathrm{pu})$, spinal cord $(\mathrm{Tl}=780.2$ $\mathrm{ms} ; \mathrm{T} 2=101.6 \mathrm{~ms}$; proton density $=58.7 \mathrm{pu})$, normal disc $(\mathrm{T} 1=1164.9 \mathrm{~ms} ; \mathrm{T} 2=101.9 \mathrm{~ms} ;$ proton density $=78.9$ pu$)$, intermediately hydrated $\operatorname{disc}(\mathrm{Tl}=723 \mathrm{~ms} ; \mathrm{T} 2=66.8 \mathrm{~ms}$; proton density $=60.8 \mathrm{pu})$, desiccated disc $(\mathrm{T} 1=554.4 \mathrm{~ms} ; \mathrm{T} 2=55.6 \mathrm{~ms} ;$ proton density $=47.6 \mathrm{~ms})$, and vertebral body $(\mathrm{T} 1=515.3 \mathrm{~ms} ; \mathrm{T} 2=100.8 \mathrm{~ms}$; proton density $=91.1 \mathrm{pu})$. Comparisons among the mean $\mathrm{T} 1, \mathrm{~T} 2$, and proton density values showed significant differences between different spinal levels (cervical, dorsal, lumbar, and sacral) for CSF (proton density), spinal cord (T2 and proton density), normal disc (T1, T2, and proton density), and vertebral bodies ( $\mathrm{T} 1$ and proton density). Significant differences were found among mean $\mathrm{T} 1, \mathrm{~T} 2$, and proton density values of normal, intermediately hydrated, and desiccated discs.

CONCLUSIONS: Measurements can be easily obtained on SyMRI and correlated with previously published values obtained using conventional relaxometry techniques.

ABBREVIATIONS: $\mathrm{PD}=$ proton density; pu = percentage units

M R imaging provides excellent soft-tissue contrast. However, this information is qualitative; on T1WI or T2WI, the absolute intensity has no physical unit and diagnosis relies on relative contrast differences between adjacent tissues in the image. ${ }^{1}$ Nevertheless, quantitative imaging is rapidly gaining importance

Received August 28, 2017; accepted after revision December 12.

From the Division of Diagnostic and Interventional Neuroradiology (M.D.-P., J.B., A.F., K.-O.L., M.I.V.), Geneva University Hospitals and Faculty of Medicine of Geneva, Geneva, Switzerland; Department of Radiology (M.D.-P.), University Hospital Marqués de Valdecilla-Instituto de Investigación Sanitaria Valdecilla, Santander, Spain; and Division of Radiology (B.M.A.D., S.B.), Geneva University Hospitals, Geneva, Switzerland.

Please address correspondence to Maria Isabel Vargas, MD, Geneva University Hospitals, Division of Diagnostic and Interventional Neuroradiology of Geneva University Hospitals, Department of Radiology and Medical Informatics, Rue

Gabrielle-Perret-Gentil 4, 1211 Genève 14,

Switzerland; e-mail: maria.i.vargas@hcuge.ch

$\equiv$ Indicates article with supplemental on-line tables.

http://dx.doi.org/10.3174/ajnr.A5566 as a complement to qualitative sequences, such as diffusion-based sequences, perfusion imaging, and MR spectroscopy.

Relaxometry is a quantitative method that allows the absolute quantification of MR imaging parameters such as T1 and T2 relaxation times as well as proton density (PD). This technique is nevertheless very time-consuming and requires $\mathrm{T} 1$ and $\mathrm{T} 2$ quantifications to be performed separately through different types of sequences that were not feasible from a practical point of view in a clinical routine setup. ${ }^{2}$ These sequences included inversion recovery $^{3}$ for $\mathrm{T} 1$ measurements or techniques derived from modifications to the Carr-Purcell Meiboom-Gill sequence, considered the criterion standard for the measurement of T2 relaxation time. ${ }^{4,5}$

Several methods are currently being developed to perform quantitative MR imaging techniques in a more efficient manner. MR fingerprinting consists of a pseudorandomized variation of the sequence parameters (flip angle, TR, and $k$-space trajectory), thus producing a unique signal evolution or "fingerprint" for each tissue. ${ }^{6}$ Following acquisition, the process involves a pattern- 
identification algorithm to link the obtained fingerprints to those stored in a predefined dictionary. ${ }^{7}$ Recent studies aim to differentiate common types of adult intra-axial brain tumors ${ }^{8}$ or to perform quantitative abdominal imaging ${ }^{9}$ using this method.

Another MR imaging quantitative method is the SyntAc sequence (based on the QRAPMASTER sequence, ${ }^{1}$ SyntheticMR, Linköping, Sweden). This is the technique we have chosen for our study because it is more readily available and is already approved by the Conformité Européenne (CE) and FDA. In this method, physical properties (PD, longitudinal [T1] and transverse [T2] relaxation rates, and correction for $\mathrm{B} 1$-inhomogeneities) are calculated using a single, multiecho, saturation recovery TSE sequence. ${ }^{10}$ Subsequently, any "synthetic" T1WI or T2WI can be achieved from the absolute parameters. ${ }^{1}$

Common advantages of these 2 methods for brain imaging are reduced acquisition time ${ }^{6}$ and the possibility of performing automatic tissue segmentation and volume estimation. ${ }^{11}$ This has already been used for the evaluation of demyelinating diseases. ${ }^{10,12}$

The recent study published by Tanenbaum et $\mathrm{al}^{13}$ compared synthetic-versus-conventional MR imaging for routine neuroimaging, concluding that synthetic MR imaging quality was comparable with conventional sequences despite artifacts being more common in the synthetic FLAIR sequence, as previously reported. ${ }^{10,14}$ This shortcoming was also reported with MR fingerprinting methodology. ${ }^{15}$

The goal of this study was to measure $\mathrm{T} 1$ and $\mathrm{T} 2$ relaxation times, as well as PD values, of normal structures included in normal spine MR imaging (CSF, spinal cord, healthy-versus-desiccated intervertebral disc, and vertebral body) using Synthetic MRI (SyMRI) 8.0 software (SyntheticMR) (this method has already been used for quantifying relaxation times in the brain, but, as far as we know, it has not been used in the spinal region). Furthermore, we aimed to compare our values with those found in the literature from studies using relaxometry.

\section{MATERIALS AND METHODS \\ Participants}

Thirty-two nonconsecutive patients (17 males; 15 females; mean age, 55.47 years; range, 17-85 years) undergoing spine MR imaging studies ( 10 cervical, 5 dorsal, 17 lumbosacral) at our institution (Geneva University Hospitals) in 2016 were included in this study.

All subjects met the following criteria: 1 ) a synthetic MR image added to our standard imaging protocol when time constraints allowed it, and 2) no history of neoplastic conditions, radiation therapy, or multiple sclerosis.

The study was approved by our institutional review board, and informed consent was waived.

\section{Image Acquisition}

All studies were performed on a $1.5 \mathrm{~T}$ Ingenia scanner (Philips Healthcare) using a 52-channel spine coil. The imaging protocol included quantitative sagittal MR imaging and the conventional MR imaging sequences used in our center, which include at least conventional 2D sagittal spin-echo T1-weighted, FSE T2weighted, and STIR sequences.

A multiple-dynamic multiple-echo sequence (SyntAc) was modified $^{1}$ for spine imaging. Parameters were the following: sagittal orientation, FOV $=200 \times 321 \mathrm{~mm}$, acquisition (reconstruction) voxel size $=0.89 \times 1.48 \mathrm{~mm}^{2}\left(0.71 \times 0.72 \mathrm{~mm}^{2}\right), 15$ contiguous slices of $4-\mathrm{mm}$ thickness, $\mathrm{TE}=11 / 100 \mathrm{~ms}, \mathrm{TR}=2485 \mathrm{~ms}$, TSE factor $=12$, sensitivity encoding acceleration factor $=2$. The acquisition time of the synthetic sequence was 5 minutes 40 seconds.

This sequence generates 8 different contrasts ( 2 different TEs and 4 different TIs), which are then processed by SyMRI 8.0 software to obtain quantitative T1 (millisecond), T2 (millisecond), and PD (pu) maps. The PD maps are measured in percentage units (pu), in which $100 \%$ is the value of brain CSF that is used for calibration. Because fat has a higher signal intensity than CSF, it is displayed with a PD value above $100 \mathrm{pu}(0-108 \mathrm{pu}$ if $\mathrm{T} 1>1100$ $\mathrm{ms}$ and $0-120 \mathrm{pu}$ if $\mathrm{T} 1<1100 \mathrm{~ms}$ ). The dynamic ranges of $\mathrm{T} 1$ and T2 values are 300-4300 and 20-2000 ms, respectively (this information was extracted from the software manual ${ }^{16}$ ).

These maps were then saved in DICOM format and analyzed in OsiriX Imaging Software (Version 7.0.3; http://www.osirixviewer.com).

\section{Radiologic Assessment}

All images were analyzed by a neuroradiologist and a fellow in neuroradiology on standard radiologic workstations connected to the PACS. A consensus was reached in the choice of representative normal-intensity areas for CSF, spinal cord, intervertebral disc, and vertebral body on each study (based on the conventional MR imaging sequences). We subsequently placed an ROI on these areas and measured representative T1 (millisecond), T2 (millisecond), and PD (pu) of each spinal element, carefully avoiding partial volumes (Figs 1 and 2).

The cervical cord was assessed at the $\mathrm{C} 2$ and C7 levels. The dorsal spinal cord was assessed on an upper and lower level around $\mathrm{T} 2$ and $\mathrm{T} 10$, respectively. In the lumbar spine, a single ROI was placed on the conus medullaris. We placed the ROI in the center of the spinal cord, including both gray and white matter, to elude a possible partial volume effect.

Two ROIs were placed in each spinal region for CSF, also on the highest and lowest possible positions, carefully avoiding descending nerve roots and flow artifacts. In the cervical studies, the upper ROI was placed in the cisterna magna, and the lower one, at the level of C7. In the dorsal studies, ROIs were placed at levels similar to the ones used for the spinal cord. In the lumbar studies, one ROI was placed at the level of the conus medullaris, and another, around S1.

All cervical, dorsal, and lumbar vertebrae fully within the FOV were included, as well as the first sacral vertebra. ROIs were placed on the anterior part of the vertebral body to avoid vascular artifacts, cortical bone, partial volume effects, and areas with signal abnormalities (eg, hemangiomas) on conventional MR imaging sequences. We excluded 3 fractured vertebrae and 2 with metallic artifacts.

All cervical, dorsal, and lumbar discs that were completely visualized were evaluated, excluding 1 with artifacts secondary to instrumentation. Intervertebral discs were measured and assigned a status of clearly normally hydrated (equivalent to a grade I of the Pfirrmann scoring ${ }^{17}$ ), intermediate signal (grades II or 

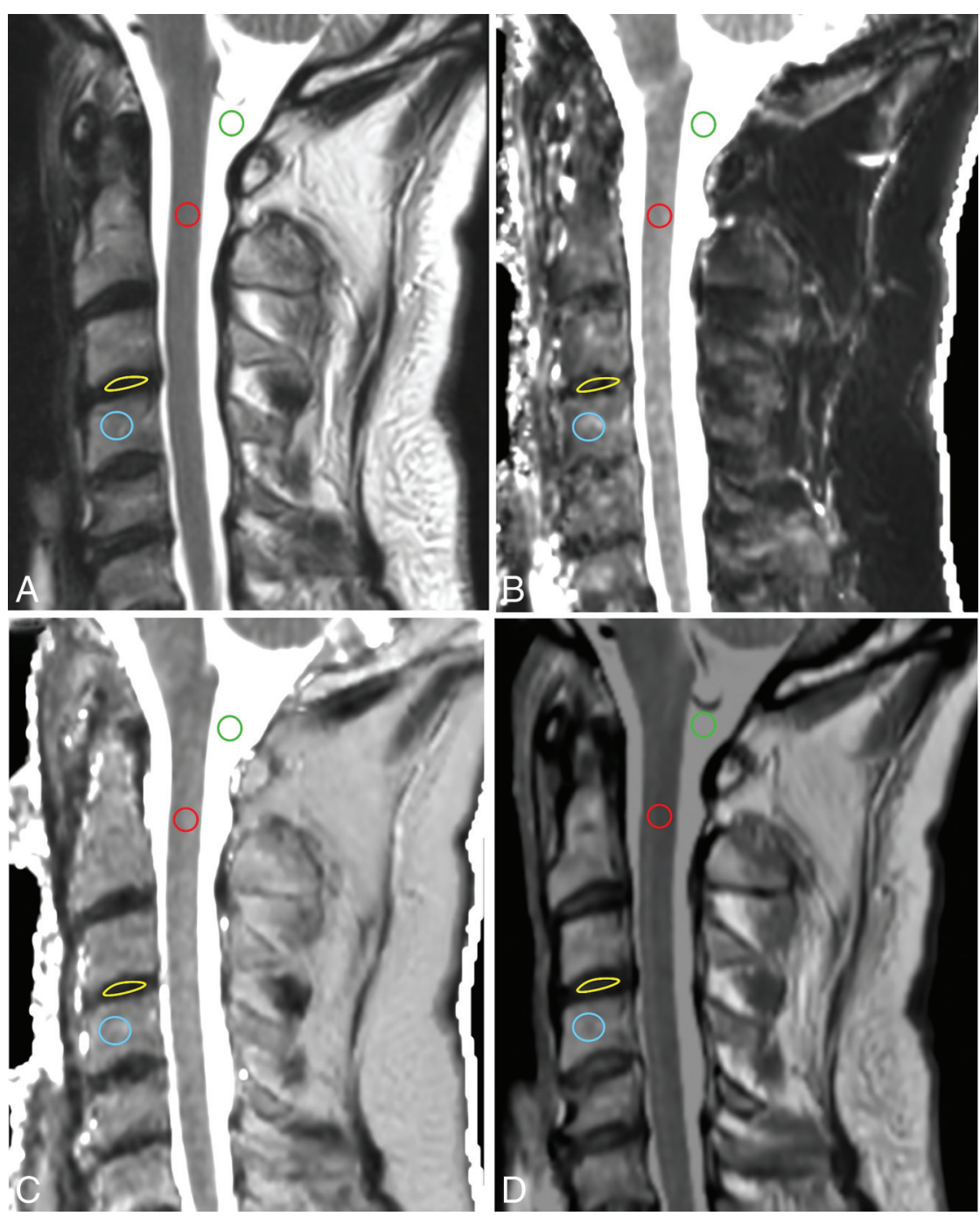

FIG 1. Conventional T2WI (A) and quantitative T1 (B), T2 (C), and PD (D) maps in a cervical study. ROIs are placed in the CSF (green), spinal cord (red), intervertebral disc (yellow), and vertebral body (blue).

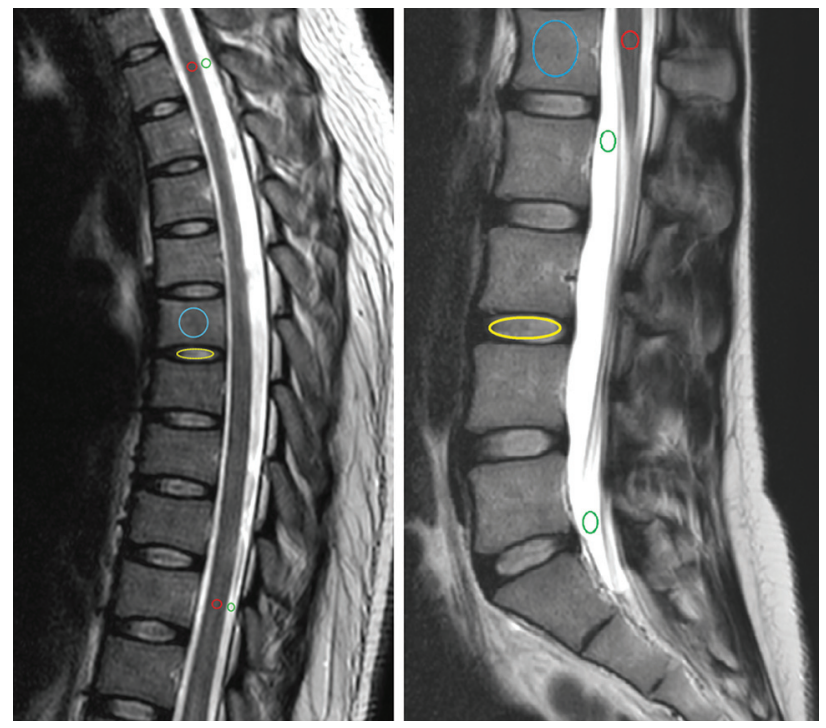

FIG 2. Conventional T2WI in a dorsal and lumbar study, showing how ROIs are placed in the CSF (green), spinal cord (red), intervertebral disc (yellow, only 1 example), and vertebral body (blue, only 1 example).
III), or desiccated (grades IV or V) by visual assessment. The intermediate signal status included discs that were neither clearly normally hydrated nor clearly desiccated. Discs showing complete loss of height were not included.

These ROIs were originally placed on the sagittal T2-weighted image and copied and pasted into the sagittal synthetic T1, T2, and PD maps; their mean value, $\mathrm{SD}$, and area were calculated.

\section{Validity of Quantification Measurements}

To verify whether the quantification with our modified SyMRI sequence with a relatively low TR (2485 ms) was correct, we compared the $\mathrm{T} 1, \mathrm{~T} 2$, and $\mathrm{PD}$ values obtained on a set of gel tubes composed of different agar and gadolinium concentrations measured with the SyMRI sequence with a TR of $6000 \mathrm{~ms}$ (and we used this technique as the criterion standard). Apart from TR, all parameters were kept identical. The acquisition time of this sequence was 11 minutes 20 seconds, which precludes its use in clinical routine. We also added an idealized model with mineral water to the FOV to include a compartment with high $\mathrm{T} 1$ and $\mathrm{T} 2$ values.

\section{Statistical Analysis}

Means and standard errors of the mean of T1, T2, and PD values for CSF, spinal cord, discs (normal, desiccated, and intermediate hydration), and vertebral bodies across all spinal levels were calculated. A 1-sample Kolmogorov-Smirnov test was used to investigate whether continuous variables were normally distributed across groups (spinal region or disc hydration status). If normal distribution of the data was confirmed in all groups, differences between group mean values were investigated by 1-way ANOVA with age as a covariate using a model with type III sum of squares. Pair-wise comparisons were subsequently performed by the least significant difference method. If data were not normally distributed in at least 1 group, differences among group median values were investigated by the nonparametric Kruskal-Wallis $\mathrm{H}$ test. The Mann-Whitney $U$ test was used for pair-wise comparisons in this case.

Correlation coefficients $(R)$ were calculated, and linear regression was performed between the values obtained with the SyMRI sequence used in the study ( $\mathrm{TR}=2485 \mathrm{~ms}$ ) and those obtained by the criterion standard ( $\mathrm{TR}=6000 \mathrm{~ms})$.

A .05 two-tailed significance level was used for all tests. Statistical analysis was performed with the Statistical Package for the Social Sciences, Version 22 (IBM, Armonk, New York). ${ }^{18}$ 


\section{RESULTS}

Table 1 shows the linear regression and the correlation coefficients obtained for T1, T2, and PD values measured with our SyMRI sequence and with a sequence with a long TR (6000 ms) used as the criterion standard. Correlation was high for T1 and T2 values $(R=1.00$ for both) and moderately high for PD values $(R=0.57)$. The slope of the line of best fit was close to 1 in all 3 analyses (1.06, 0.96, and 0.94 for T1, T2, and PD, respectively).

Descriptive statistics with means and 95\% confidence intervals of the mean of T1, T2, and PD measured values of CSF, spinal cord, normal discs, intermediately hydrated discs, desiccated discs, and vertebral bodies for all spinal levels are shown in Table 2.

\section{Comparison of Spinal Levels}

The Kolmogorov-Smirnov test showed that only T1 and PD values for CSF were not normally distributed in any of the spinal levels (cervical, dorsal, lumbar, and sacral) $(P<.05)$. The nonparametric Kruskal-Wallis $\mathrm{H}$ test was therefore used to compare medians of spinal levels in these 2 variables. The distribution of

Table 1: Validity of quantification measurements

\begin{tabular}{llc}
\hline $\begin{array}{c}\text { Range of Values } \\
\text { (Water Bottle) }\end{array}$ & $\begin{array}{c}\text { Linear Regression } \\
\text { Equation }\end{array}$ & $\boldsymbol{R}$ \\
\hline $\mathrm{T} 1,300-822(2819)$ & $\mathrm{y}=1.06 \mathrm{x}-40.1$ & 1.00 \\
$\mathrm{~T} 2,41-255(1929)$ & $\mathrm{y}=0.96 \mathrm{x}-4.72$ & 1.00 \\
$\mathrm{PD}, 41.8-131.4(100.9)$ & $\mathrm{y}=0.94 \mathrm{x}+7.44$ & 0.57 \\
\hline
\end{tabular}

Note:- Linear regression and correlation coefficients between $\mathrm{T}, \mathrm{T} 2$ and PD values measured with the SyntheticMR sequence with TR $6000 \mathrm{~ms}$ (criterion standard) and the sequence used in this study (TR 2385 ms). Range of values investigated is indicated in first column.

Table 2: Normal values of CSF, spinal cord, discs and vertebral bodies

\begin{tabular}{lccc}
\hline & $\mathrm{T1}(\mathrm{ms})$ 95\% Cl & T2 (ms) 95\% Cl & PD (pu) 95\% Cl \\
\hline CSF $(n=64)$ & $4273.4(4263.8-4283.1)$ & $1577.6(1501.2-1654.0)$ & $107.5(107.2-107.8)$ \\
Spinal cord $(n=45)$ & $780.2(753.6-806.9)$ & $101.6(98.7-104.6)$ & $58.7(56.9-60.6)$ \\
Normal disc $(n=64)$ & $1164.9(1071.1-1258.7)$ & $101.9(94.2-109.5)$ & $78.9(76.0-81.9)$ \\
Intermediate disc $(n=29)$ & $723(677.3-768.6)$ & $66.8(62.6-71.1)$ & $60.8(58.2-63.4)$ \\
Desiccated disc $(n=144)$ & $554.4(536.7-572.0)$ & $55.6(54.2-57.0)$ & $47.6(46.2-49.0)$ \\
Vertebral body $(n=260)$ & $515.3(506.0-524.6)$ & $100.8(99.5-102.2)$ & $91.1(89.6-92.5)$ \\
\hline
\end{tabular}

Note:-Means and $95 \% \mathrm{Cl}$ of the mean of $\mathrm{T} 1, \mathrm{~T} 2$ and PD measured values of CSF, spinal cord, discs, and vertebral bodies for all spinal levels.

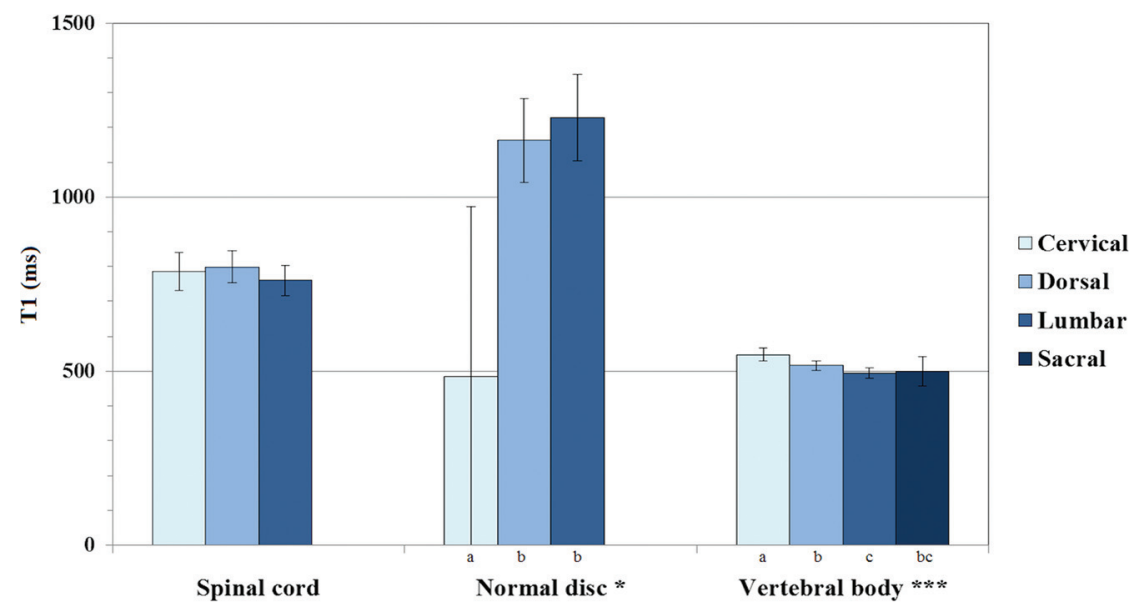

FIG 3. ANOVA results with comparison of estimated marginal means of $T 7$ values for spinal cord, normal discs, and vertebral bodies across spinal levels, assuming fixed ages of 56.2, 36.9, and 56.4 years, respectively (single asterisk indicates $P<.05$; double asterisks, $P<.01$; triple asterisks, $P<$ .001). Significant pair-wise differences between spinal levels are indicated by a different letter assigned to each level. all the other variables was not significantly different from normality in all spinal levels, which allowed comparison of means by ANOVA.

We did not find a significant difference among different spinal levels in spinal cord T1 values $(P=.456)$. The T1 value of normal discs across spinal levels was significantly different, with cervical spine discs showing the largest difference in relation to dorsal and lumbar discs $(P<.05)$. There was an even more significant effect of spinal level on the T1 value of vertebral bodies $(P<.001)$. These results are summarized in Fig 3.

Spinal level had a significant effect on T2 signal intensity of the spinal cord and normal discs $(P<.05$ and $P<.001$, respectively). Overall, the $\mathrm{T} 2$ value of normal discs increased progressively from the cervical to the lumbar region. The T2 value of vertebral bodies was not significantly affected by spinal level $(P=.370)$ (Fig 4$)$. Similarly, CSF T2 values did not show a significant difference among spinal levels $(P=.081)$ (Fig 5$)$.

PD values of spinal cord, normal discs, and vertebral bodies were significantly affected by spinal level $(P<.01, P<.001$, and $P<.001$, respectively). PD values of the spinal cord and vertebral bodies tended to decrease from cervical to lumbar, whereas PD values of discs tended to increase (Fig 6).

Medians of CSF T1 values were not significantly different across spinal levels $(P=.234)$. Despite the noticeable similarity of CSF PD values in the 4 different spinal levels, a significant difference was found among medians $(P<.05)$ (Table 3$)$.

\section{Comparison of Disc Hydration Statuses}

Means of disc T1 values were strongly affected by disc hydration status $(P<$ $.001)$, with progressively lower values as the disc water content decreased (Fig 7). The same trend was found on the nonparametric test results with disc T2 and $\mathrm{PD}$ values significantly decreasing as disc hydration declined $(P<.001)$ (Table 4).

\section{DISCUSSION \\ CSF}

Our results for CSF mean relaxation times are comparable with those found in the literature. Krauss et $\mathrm{al}^{19}$ showed $\mathrm{T} 1, \mathrm{~T} 2$, and PD values very similar to ours on the charts displaying their phantom measurements with the QRAPMASTER technique. Using the same technique, Warntjes et $\mathrm{al}^{1}$ obtained $\mathrm{T} 1$ and $\mathrm{T} 2$ values of $3940 \mathrm{~ms}$ and 1910 ms, respectively, and PD values of $1029 \mathrm{pu}$, specifying that "the PD of pure water at $37^{\circ} \mathrm{C}$ corresponds to 1000 " and arguing that the proton density of CSF appeared "somewhat high, possibly due to flow-effects or diffusion." In a review article from 2017, Bojorquez et $\mathrm{al}^{4}$ reported the following CSF T1 values in 


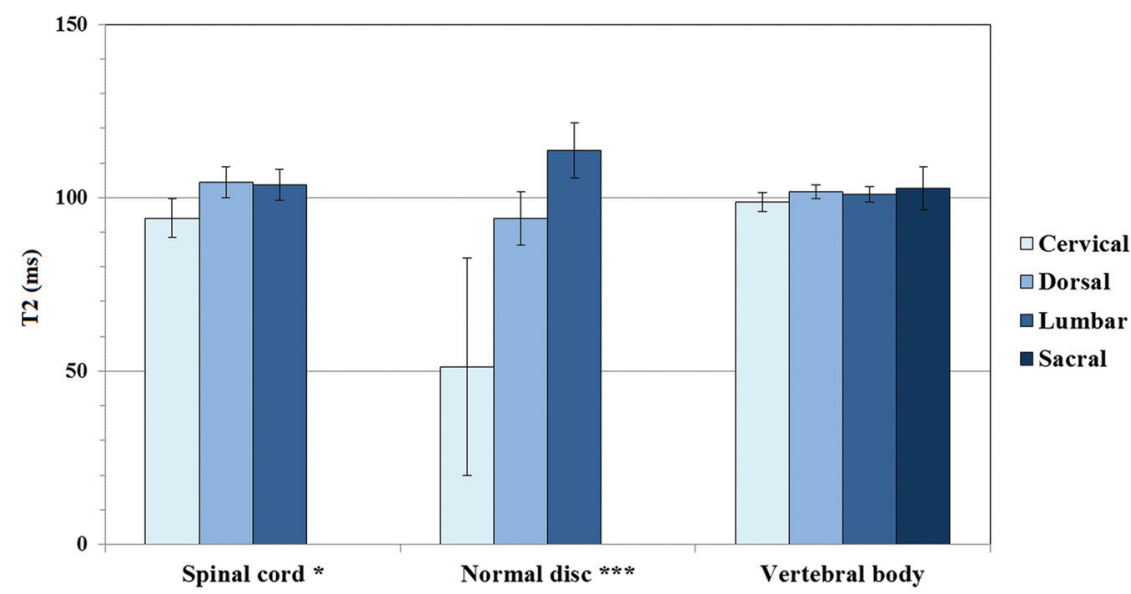

FIG 4. ANOVA results with comparison of estimated marginal means of $T 2$ values for spinal cord, normal discs, and vertebral bodies across spinal levels, assuming fixed ages of 56.2, 37.2, and 56.4 years, respectively (single asterisk indicates $P<.05$; double asterisks, $P<.01$; triple asterisks, $P<$ $.001)$. Significant pair-wise differences between spinal levels are indicated by a different letter assigned to each level.

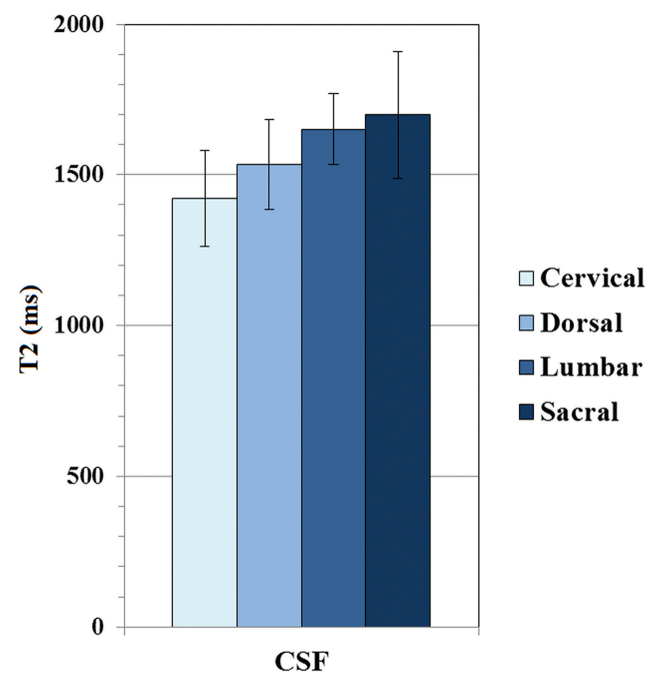

FIG 5. ANOVA results with comparison of estimated marginal means of T2 values for CSF across spinal levels assuming a fixed age of 55.5 years. There was no significant difference among spinal levels.

the literature: $4391 \pm 545 \mathrm{~ms},{ }^{20} 3817 \pm 424 \mathrm{~ms},{ }^{5} 4522 \pm 417$ $\mathrm{ms},{ }^{20} 6873 \mathrm{~ms}$, and $4184 \mathrm{~ms}^{21}$

We found significant differences in CSF values among spinal levels only in PD, despite the medians being very similar. This finding was unexpected and could potentially represent a falsepositive result. However, another possible explanation for this difference is that, due to our slice thickness ( $4 \mathrm{~mm})$, partial volume could have been introduced from the nerve roots of the cauda equina at the sacral level, therefore lowering the measured CSF PD values. Because this second explanation is not immediately apparent from the analysis of the median values, we provide means of PD CSF values by spinal level: $107.86 \pm 0.36$ for the cervical spine, $107.97 \pm 0.09$ for the dorsal spine, $107.35 \pm 1.31$ for the lumbar spine, and $106.67 \pm 2.06$ for the sacral spine.

$\mathrm{T} 1$ and PD values for CSF were not normally distributed. As mentioned in the "Materials and Methods" section, we found an upper limit of 108 for PD and 4300 for T1 with this technique, which was produced by the software and could be considered a systematic bias, as we further discuss in the limitations in the "Discussion." Conversely, all $\mathrm{T} 2$ values were within the dynamic range of measurement.

Limitations to CSF measurements were the presence of flow artifacts, which are slightly more prominent than those usually encountered in routine imaging, especially in the T2 and PD maps, as previously reported. ${ }^{10}$

\section{Spinal Cord}

$\mathrm{T} 1$ relaxation times in the spinal cord reported by Smith et $\mathrm{al}^{22}$ were $863 \pm 23$ $\mathrm{ms}$ and $900 \pm 17 \mathrm{~ms}$ for white matter and $972 \pm 36 \mathrm{~ms}$ for gray matter using a $3 \mathrm{~T}$ MR imaging system. Massire et $\mathrm{al}^{23}$ reported $1189 \pm 42 \mathrm{~ms}$ for white matter and $1313 \pm 51 \mathrm{~ms}$ for gray matter. Both publications reported higher values than our mean of $780 \pm 26.6 \mathrm{~ms}$. This difference is probably because their study was performed at $3 \mathrm{~T}$ and $7 \mathrm{~T}$ and relaxometry measures (especially T1) are dependent on field strength.

$\mathrm{T} 2$ relaxation times reported in the literature are $73 \mathrm{~ms}$ for white matter and $76 \mathrm{~ms}$ for gray matter, ${ }^{22}$ which differ from our $\mathrm{T} 2$ mean value of $101.6 \pm 2.9 \mathrm{~ms}$. Lu et $\mathrm{al}^{5}$ reported $\mathrm{T} 2$ values for brain gray matter at $1.5 \mathrm{~T}$, ranging from 81 (putamen) to $99 \mathrm{~ms}$ (cortex). We did not find any previously reported PD values for the spinal cord.

Although the absolute values did not show large variations, we found that the cervical region had a significantly different T2 value from the thoracic and lumbar regions (94.1 versus 104.4 and $103.6 \mathrm{~ms}$, respectively). The same difference was found in PD values (63.3 versus 58.7 and $55.7 \mathrm{pu}$, respectively). No significant differences were found for $\mathrm{T} 1$ values. This may relate to the normal anatomy of the spinal cord because the configuration of the gray matter and gray/white matter ratio varies along spinal cord regions, depending on the neural requirements of a given region. ${ }^{24}$ There is also variation in the total number of tracts (which decreases from cervical to lumbar, as more and more nerve projections arise), as well as in the cross-sectional area of the cord (which increases at the cervical and lumbar enlargements). These 2 features determine variations in the density of spinal tracts along different segments of the cord.

We believe SyMRI could prove to be a good quantitative MR imaging technique for the assessment of the spinal cord. T1, T2, and PD measurements have the potential to be used as biomarkers of both myelin and axonal integrity. A promising application could therefore be to evaluate the degree of spinal cord integrity in posttraumatic or demyelinating lesions. Further studies would be needed to corroborate the variations we found among spinal levels and clarify the possible causes. Recently, the role of T1, T2, PD, and myelin measurements has been analyzed in different neurologic conditions in the brain, ${ }^{25}$ and we believe it could potentially be directly translated into spinal cord conditions. Moreover, although outside of the scope of this study, myelin water fraction can be extracted from SyMRI T2 measures. ${ }^{26}$ Preliminary studies 


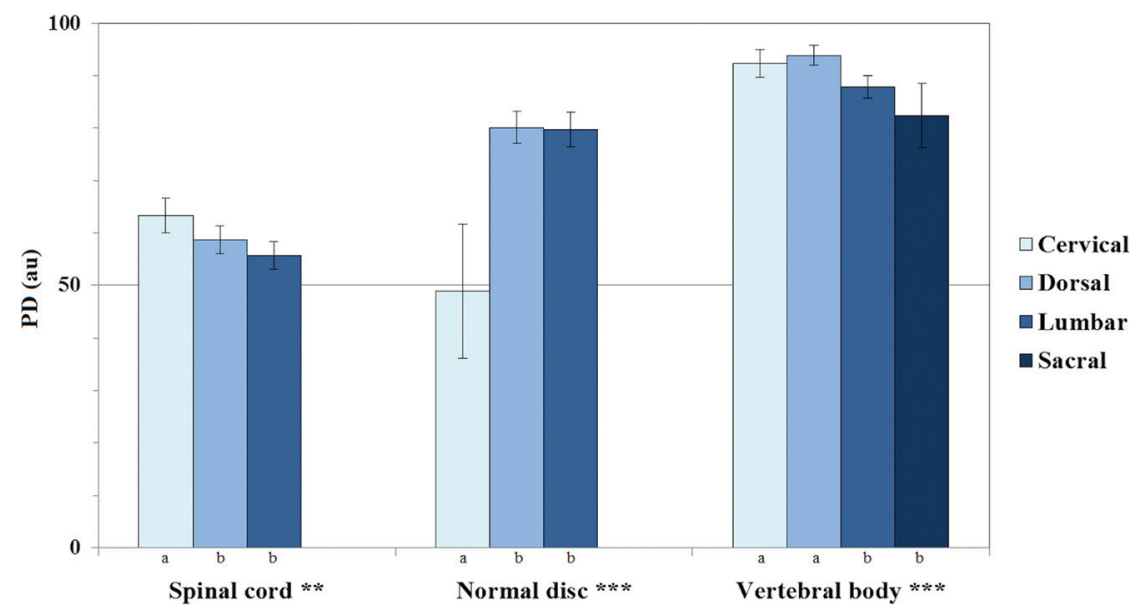

FIG 6. ANOVA results with comparison of estimated marginal means of PD values for spinal cord, normal discs, and vertebral bodies across spinal levels, assuming fixed ages of 56.2, 37.2, and 56.4 years, respectively (single asterisk indicates $P<.05$; double asterisks, $P<.01$; triple asterisks, $P<$ .001). Significant pair-wise differences between spinal levels are indicated by a different letter assigned to each level.

Table 3: Results of the Kruskal-Wallis $\mathrm{H}$ test with $\mathrm{T1}$ and PD median values for CSF across spinal levels

\begin{tabular}{lccccc}
\hline & Cervical & Dorsal & Lumbar & Sacral & $\boldsymbol{P}$ \\
\hline TI CSF & 4287.7 & 4285.5 & 4289.6 & 4294.3 & .234 \\
PD CSF & $108.0^{\mathrm{a}, \mathrm{b}}$ & $108.0^{\mathrm{a}}$ & $108.0^{\mathrm{b}}$ & $108.0^{\mathrm{a}, \mathrm{b}}$ & $<.05$ \\
\hline
\end{tabular}

Note:-Significant pairwise differences between spinal levels are indicated by a different letter (a, b) assigned to each level.

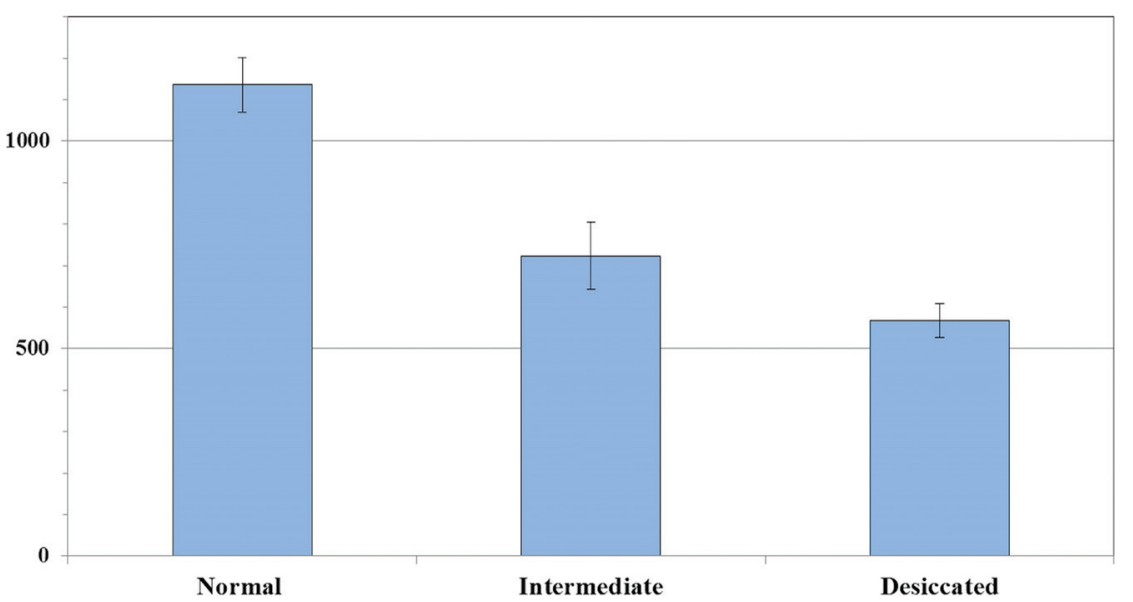

FIG 7. ANOVA results with comparison of estimated marginal means of $T 7$ values for normal, intermediately hydrated, and desiccated discs, assuming a fixed age of 55.9 years, which shows a significant effect of hydration status $(P<.001)$. All pair-wise comparisons also show a significant difference among groups.

Table 4: Results of the Kruskal-Wallis $\mathrm{H}$ test with median $\mathrm{T} 2$ and PD values for discs across the 3 hydration status

\begin{tabular}{lcccc}
\hline & Normal & Intermediate & Desiccated & $\boldsymbol{P}$ \\
\hline T2 disc & $95.5^{\mathrm{a}}$ & $66.2^{\mathrm{b}}$ & $54.0^{\mathrm{c}}$ & $<.001$ \\
PD disc & $80.9^{\mathrm{a}}$ & $58.0^{\mathrm{b}}$ & $47.8^{\mathrm{c}}$ & $<.001$ \\
\hline
\end{tabular}

Note:-Significant pairwise differences between hydration status are indicated by a different letter (a, b, c) assigned to each level.

applied to multiple sclerosis have already shown interesting results with this technique, which seems to be more sensitive than longitudinal and transverse relaxation rates (R1 and R2, respectively) and PD for plaque assessment, even though some changes are already seen with these parameters. ${ }^{27}$

\section{Discs}

We have found significant differences among mean T1, T2, and PD values of normal, intermediately hydrated, and desiccated discs, confirming the formerly described negative relationship between relaxation time (T1, T2) and disc-degenerative grade. ${ }^{28} \mathrm{~T} 1$ relaxation time has been shown to decrease with disc degeneration ${ }^{29}$ and herniation. ${ }^{30}$ T2 relaxation times are related to disc biochemical composition in that decreased T2 values represent decreased disc water content and proteoglycan $\operatorname{loss}^{31}$ or replacement of the nucleus by degenerative tissue, which has lower T2 values. $^{32}$

Our results also showed significant differences in disc values among different spinal levels, with lower T1, T2, and $\mathrm{PD}$ values in the cervical spine compared with the thoracic or lumbar levels. This finding agrees with previous biochemistry reports of lower water content in cervical discs compared with lumbar discs. $^{33}$

Another possible explanation for our significantly lower values in cervical discs could be related to the ROI areas, which were smaller in that region. This feature may have affected the proportion of annulus fibrosus or its analogous fibrous structure, the intranuclear cleft versus the nucleus pulposus. As reported by Stelzeneder et al in 2012, ${ }^{34}$ the annulus fibrosus has significantly lower values compared with the nucleus pulposus. Because the reduction in cervical disc areas is mainly at the expense of nucleus pulposus, this finding could be another reason for their decreased T1, T2, and $\mathrm{PD}$ values.

Limitations to our conclusions in disc analysis could be because we established disc hydration status by agreed visual assessment and the age range is wide in our population (17 to 81 years), possibly explaining the large variability of the values obtained for discs (and vertebral bodies).

Disc degeneration is a condition reported daily on spine examinations, and SyMRI could help establish universal cutoffs (settled by spinal level) for staging it.

\section{Vertebral Bodies}

Our mean values of T1 and T2 mean relaxation times are consistent with those found in the literature. In the Bojorquez et al review, ${ }^{4}$ the values obtained for T1 vary between $106 \pm 4 \mathrm{~ms}^{35}$ and 
$586 \pm 73 \mathrm{~ms},{ }^{3}$ and from 40 to $160 \mathrm{~ms}$ for T2. ${ }^{36}$ Le Ster et al ${ }^{37}$ reported $\mathrm{T} 1$ values of $621 \mathrm{~ms}$.

T2 relaxation times did not show significant differences among spinal levels, but T1 values significantly decreased from cervical to lumbar (from 547.1 to $495.1 \mathrm{~ms}$ ) as did PD values (from 92.3 to $82.4 \mathrm{pu}$ ). Because bone marrow is characterized by large variations in $\mathrm{T} 1$ relaxation times between water and fat compartments, ${ }^{38}$ the differences found may be due to normal anatomic changes in the proportion of fatty marrow along the spine (which increases from C3 to L5). ${ }^{39}$

A potential application of determining T1, T2, and PD values of vertebral bodies could be in the detection of bone marrow infiltrative conditions, such as hematologic malignancies, by providing a quantitative tool for imaging assessment in situations in which it is difficult to visually confirm and demarcate pathologic changes. However, consideration must be given to the high variability relating to vertebral body composition among healthy individuals because it depends on different factors such as age, with conversion from red to yellow marrow, or the effect of hematocrit levels.

The On-line Table outlines previously published measured values with conventional relaxometry techniques for CSF, spinal cord, disc, and bone marrow.

Our study has some limitations, some of which have already been mentioned above. Another limitation is the potential bias introduced by manually performing some operations such as ROI selection. For spinal cord measurements, atlas-based techniques are available that could potentially have allowed gray-white matter distinction.

Nonconsecutive selection of patients may have introduced some bias. We nevertheless believe that this is minimized because there were no criteria in selecting patients for the study other than time constraints allowing the synthetic sequence to be performed. Taking this into account, all patients in whom this sequence was performed, within the time considered, took part in the study; this feature made the selection consecutive in this subgroup of patients.

We probably also have some limitations in accurately quantifying elements with high T1 and PD because our sequence has a relatively low TR (2485 ms). This limitation means that complete relaxation of high $\mathrm{T} 1$ structures is not fulfilled during the sequence; therefore, signal can be somehow saturated. Our attempt to verify the validity of quantification due to our short TR showed a high correlation of values for a T1 range of 300-822 ms as well as in the idealized model with T1 of $2819 \mathrm{~ms}$. However, we did not compare the validity of the measurements with higher $\mathrm{T} 1$ as is the case with CSF; this difference may have biased the results obtained in this compartment due to the low TR.

Moreover, when we analyzed structures with small dimensions, such as the spinal cord, our quantitative values could have partial volume effects due to our slice thickness of $4 \mathrm{~mm}$. This could be an explanation for the higher T2 values found in the spinal cord compared with previously published values in the literature.

Another limitation of our study is the lack of comparison among the values obtained with an internal criterion standard. These values would be more meaningful if compared with quan- titative measures using conventional techniques in the same patients. Unfortunately, it is difficult in practice to add conventional sequences to the imaging protocol due to the additional acquisition time needed. Similarly, conventional T1 and T2 quantitative mapping techniques were not used for the agar phantom experiments. We are aware that SyMRI with a TR of 6000 is not a true criterion standard. However, quantification with SyMRI (TR ranging from 2900 to $5000 \mathrm{~ms}$ ) has been validated in several previous publications. ${ }^{1,11,19,40-42}$

\section{CONCLUSIONS}

$\mathrm{T} 1, \mathrm{~T} 2$, and PD measurements can be easily and rapidly obtained with SyMRI, a tool that could be readily available as a quantitative method in the spinal region, retrieving results comparable with those made previously by other quantitative techniques.

It is likely that the use of quantitative MR imaging will be implemented as part of the radiologist's every day work. Setting the reference range for relaxation times of CSF, spinal cord, vertebral bodies, and discs in healthy individuals will therefore have a straightforward application. These measurements could be used in our future daily practice as a quantitative extra aid in the radiologic assessment of spinal conditions, such as infiltration of bone marrow in hematologic disease, demyelinating disease, and spondylodiscitis. They could also play a role in the classification of disc degeneration and the choice of treatment.

\section{ACKNOWLEDGMENTS}

M.D.-P. is supported by a research contract (Contrato post-MIR López Albo 2016) from the Instituto de Investigación Sanitaria Valdecilla (IDIVAL).

\section{REFERENCES}

1. Warntjes JB, Leinhard OD, West J, et al. Rapid magnetic resonance quantification on the brain: optimization for clinical usage. Magn Reson Med 2008;60:320-29 CrossRef Medline

2. Bottomley PA, Foster TH, Argersinger RE, et al. A review of normal tissue hydrogen NMR relaxation times and relaxation mechanisms from 1-100 MHz: dependence on tissue type, NMR frequency, temperature, species, excision, and age. Med Phys 1984;11:425-48 CrossRef Medline

3. de Bazelaire CM, Duhamel GD, Rofsky NM, et al. MR imaging relaxation times of abdominal and pelvic tissues measured in vivo at 3.0 T: preliminary results. Radiology 2004;230:652-59 CrossRef Medline

4. Bojorquez JZ, Bricq S, Acquitter C, et al. What are normal relaxation times of tissues at 3 T? Magn Reson Imaging 2017;35:69-80 CrossRef Medline

5. Lu H, Nagae-Poetscher LM, Golay X, et al. Routine clinical brain MRI sequences for use at 3.0 Tesla. J Magn Reson Imaging 2005;22: 13-22 CrossRef Medline

6. Ma D, Gulani V, Seiberlich N, et al. Magnetic resonance fingerprinting. Nature 2013;495:187-92 CrossRef Medline

7. European Society of Radiology (ESR). Magnetic resonance fingerprinting: a promising new approach to obtain standardized imaging biomarkers from MRI. Insights Imaging 2015;6: 163-65 CrossRef Medline

8. Badve C, Yu A, Dastmalchian S, et al. MR fingerprinting of adult brain tumors: initial experience. AJNR Am J Neuroradiol 2017;38: 492-99 CrossRef Medline

9. Chen $Y$, Jiang $Y$, Pahwa $S$, et al. MR fingerprinting for rapid quantitative abdominal imaging. Radiology 2016;279:278-86 CrossRef Medline

10. Granberg T, Uppman M, Hashim F, et al. Clinical feasibility of syn- 
thetic MRI in multiple sclerosis: a diagnostic and volumetric validation study. AJNR Am J Neuroradiol 2016;37:1023-29 CrossRef Medline

11. West J, Warntjes JB, Lundberg P. Novel whole brain segmentation and volume estimation using quantitative MRI. Eur Radiol 2012;22: 998-1007 CrossRef Medline

12. Vågberg M, Lindqvist T, Ambarki K, et al. Automated determination of brain parenchymal fraction in multiple sclerosis. AJNR Am J Neuroradiol 2013;34:498-504 CrossRef Medline

13. Tanenbaum LN, Tsiouris AJ, Johnson AN, et al. Synthetic MRI for clinical neuroimaging: results of the Magnetic Resonance Image Compilation (MAGiC) prospective, multicenter, multireader trial. AJNR Am J Neuroradiol 2017;38:1 103-10 CrossRef Medline

14. Blystad I, Warntjes JB, Smedby O, et al. Synthetic MRI of the brain in a clinical setting. Acta Radiol 2012;53:1158-63 CrossRef Medline

15. Deshmane A, McGivney D, Badve C. Accurate synthetic FLAIR images using partial volume corrected MR fingerprinting. In: Proceedings of the Annual Meeting and Exhibition of International Society for Magnetic Resonance in Medicine, Singapore. May 7-13, 2016

16. User Manual, Version 8.0.1, SyMRI 8. Linköping: SyntheticMR AB; 2016-10-26

17. Pfirrmann CW, Metzdorf A, Zanetti M, et al. Magnetic resonance classification of lumbar intervertebral disc degeneration. Spine (Phila Pa 1976) 2001;26:1873-78 CrossRef Medline

18. IBM SPSS Statistics (for Windows) [computer program]. Version 22.0. Armonk, New York: IBM Corp; 2013

19. Krauss W, Gunnarsson M, Andersson T, et al. Accuracy and reproducibility of a quantitative magnetic resonance imaging method for concurrent measurements of tissue relaxation times and proton density. Magn Reson Imaging 2015;33:584-91 CrossRef Medline

20. Shin W, Gu H, Yang Y. Fast high-resolution T1 mapping using inversion-recovery Look-Locker echo-planar imaging at steady state: optimization for accuracy and reliability. Magn Reson Med 2009;61: 899-906 CrossRef Medline

21. Liberman $G$, Louzoun $Y$, Ben Bashat D. $T_{1}$ mapping using variable flip angle SPGR data with flip angle correction. J Magn Reson Imaging 2014;40:171-80 CrossRef Medline

22. Smith SA, Edden RA, Farrell JA, et al. Measurement of T1 and T2 in the cervical spinal cord at 3 Tesla. Magn Reson Med 2008;60:213-19 CrossRef Medline

23. Massire A, Taso M, Besson P, et al. High-resolution multi-parametric quantitative magnetic resonance imaging of the human cervical spinal cord at 7T. Neuroimage 2016;143:58-69 CrossRef Medline

24. Diaz E, Morales H. Spinal cord anatomy and clinical syndromes. Semin Ultrasound CT MR 2016;37:360-71 CrossRef Medline

25. Hagiwara A, Hori M, Yokoyama K, et al. Utility of a multiparametric quantitative MRI model that assesses myelin and edema for evaluating plaques, periplaque white matter, and normal-appearing white matter in patients with multiple sclerosis: a feasibility study. AJNR Am J Neuroradiol 2017;38:237-42 CrossRef Medline

26. Warntjes M, Engström M, Tisell A, et al. Modeling the presence of myelin and edema in the brain based on multi-parametric quantitative MRI. Front Neurol 2016;7:16 CrossRef Medline

27. Hagiwara A, Warntjes M, Hori M, et al. SyMRI of the brain: rapid quantification of relaxation rates and proton density, with synthetic MRI, automatic brain segmentation, and myelin measurement. Invest Radiol 2017;52:647-57 CrossRef Medline

28. Hwang D, Kim S, Abeydeera NA, et al. Quantitative magnetic reso- nance imaging of the lumbar intervertebral discs. Quant Imaging Med Surg 2016;6:744-55 CrossRef Medline

29. Chatani K, Kusaka Y, Mifune T, et al. Topographic differences of $1 \mathrm{H}-\mathrm{NMR}$ relaxation times $(\mathrm{T} 1, \mathrm{~T} 2)$ in the normal intervertebral disc and its relationship to water content. Spine (Phila Pa 1976) 1993;18:2271-75 CrossRef Medline

30. Boos N, Wallin A, Schmucker T, et al. Quantitative MR imaging of lumbar intervertebral disc and vertebral bodies: methodology, reproducibility, and preliminary results. Magn Reson Imaging 1994; 12:577-87 CrossRef Medline

31. Marinelli NL, Haughton VM, Muñoz A, et al. T2 relaxation times of intervertebral disc tissue correlated with water content and proteoglycan content. Spine (Phila Pa 1976) 2009;34:520-24 CrossRef Medline

32. Grunert P, Hudson KD, Macielak MR, et al. Assessment of intervertebral disc degeneration based on quantitative magnetic resonance imaging analysis: an in vivo study. Spine (Phila Pa 1976) 2014;39: E369-78 CrossRef Medline

33. Driscoll SJ, Zhong W, Torriani M, et al. In-vivo T2-relaxation times of asymptomatic cervical intervertebral discs. Skeletal Radiol 2016; 45:393-400 CrossRef Medline

34. Stelzeneder D, Welsch GH, Kovács BK, et al. Quantitative T2 evaluation at 3.0T compared to morphological grading of the lumbar intervertebral disc: a standardized evaluation approach in patients with low back pain. Eur J Radiol 2012;81:324-30 CrossRef Medline

35. Wang G, El-Sharkawy AM, Edelstein WA, et al. Measuring T2 and T1, and imaging T2 without spin echoes. J Magn Reson 2012;214: 273-80 CrossRef Medline

36. Pai A, Li X, Majumdar S. A comparative study at $3 \mathrm{~T}$ of sequence dependence of T2 quantitation in the knee. Magn Reson Imaging 2008;26:1215-20 CrossRef Medline

37. Le Ster C, Lasbleiz J, Kannengiesser S, et al. A fast method for the quantification of fat fraction and relaxation times: comparison of five sites of bone marrow. Magn Reson Imaging 2017;39:157-61 CrossRef Medline

38. Schick F, Bongers $\mathrm{H}$, Jung W, et al. Proton relaxation times in human red bone marrow by volume selective magnetic resonance spectroscopy. Appl Magn Reson 1992;3:947-63 CrossRef

39. Baum T, Yap SP, Dieckmeyer M, et al. Assessment of whole spine vertebral bone marrow fat using chemical shift-encoding based water-fat MRI. J Magn Reson Imaging 2015;42:1018-23 CrossRef Medline

40. Warntjes JB, Engström M, Tisell A, et al. Brain characterization using normalized quantitative magnetic resonance imaging. PLoS One 2013;8:e70864 CrossRef Medline

41. Engström M, Warntjes JB, Tisell A, et al. Multi-parametric representation of voxel-based quantitative magnetic resonance imaging. PLoS One 2014;9:e111688 CrossRef Medline

42. West J, Blystad I, Engström M, et al. Application of quantitative MRI for brain tissue segmentation at $1.5 \mathrm{~T}$ and $3.0 \mathrm{~T}$ field strengths. $P L O S$ One 2013;8:e74795 CrossRef Medline

43. Chen L, Bernstein M, Huston J, et al. Measurements of T1 relaxation times at 3.0 T: implications for clinical MRA. In: Proceedings of the International Society for Magnetic Resonance in Medicine 9th Scientific Meeting \& Exhibition, Glasgow, Scotland. April 21-27, 2001

44. Chen C, Huang M, Han Z, et al. Quantitative T2 magnetic resonance imaging compared to morphological grading of the early cervical intervertebral disc degeneration: an evaluation approach in asymptomatic young adults. PLoS One 2014;9:e87856 CrossRef Medline 